\title{
Interaction of Nearly-Inviscid, Multi-mode Faraday Waves and Mean Flows
}

\author{
María J. Higuera, José M. Vega, and Edgar Knobloch \\ E.T.S. Ingenieros Aeronáuticos, \\ Pza. Cardenal Cisneros 3, 28040 Madrid, Spain \\ Department of Physics, University of California, Berkeley, \\ California 94720
}

\section{Introduction}

Faraday waves [1] are gravity-capillary waves that are excited on the surface of a fluid when its container is vibrated vertically and the vertical acceleration exceeds a threshold value. These waves have received much attention in the literature both as a basic fluid dynamical problem and as a paradigm of a pattern-forming system $[2,3,4]$. Unfortunately, in the low viscosity limit, there are several basic issues that remain unresolved, particularly in connection with the generation of mean flows in the bulk. The viscous part of these flows (also called streaming flow or acoustic streaming) is driven by the oscillatory boundary layers attached to the solid walls and the free surface by well-known mechanisms first uncovered by Schlichting [5] and Longuet-Higgins [6]. This mean flow has been shown recently to affect the dynamics of the primary waves at leading order in a related, laterally vibrated system [7]. This is somewhat similar to the effect of an internal circulation on surface wave dynamics in drops [8].

The main object of this note is to present and discuss a set of asymptotically correct (as viscosity and wave steepness go to zero) coupled amplitude-mean flow (CASF) equations for multi-mode Faraday waves in containers of general cross-section. It will be seen that it is asymptotically inconsistent to ignore the streaming flow while retaining the usual cubic nonlinear terms whenever more than one surface mode is present. Symmetries of the problem, if present, limit the scope of the interaction with the mean flow; however, as the number of excited surface modes increases, the interaction becomes stronger and stronger. Despite the fact that the CASF equations include 3-D continuity and Navier-Stokeslike equations for the mean flow some qualitative properties of the solutions can be established. As an example, we consider in $\$ 4$ two-mode Faraday waves in almost-square, rectangular containers, as in the well-known experiments by Simonelli and Gollub [9] and Feng and Sethna [10].

\section{Formulation}

We consider a vertically vibrated cylindrical container of general cross-section $\Sigma$. In order to avoid the uncertainties associated with the modeling of contactline dynamics [2] and additional difficulties due to a strong singularity in the 
velocity at a moving contact line when the contact angle differs from 0 or $\pi$ [11], the contact line is assumed to be pinned to the upper edge of the vertical wall of the container, as done in some Faraday experiments [12] in order to eliminate the lateral meniscus and the associated meniscus waves. For nondimensionalization, we use the unperturbed depth, $\ell$, as characteristic length and, in order to take into account simultaneously both gravity and microgravity conditions, we use the gravity-capillary time $\left[g / \ell+\sigma /\left(\rho \ell^{3}\right)\right]^{-1 / 2}$ as the characteristic time. Here $g$ is the gravitational acceleration, $\sigma$ is the surface tension coefficient and $\rho$ is the density, all assumed to be constant. We use a Cartesian coordinate system attached to the vibrating container, with the $z=0$ plane at the unperturbed free surface, assumed to be horizontal. The governing continuity and momentum conservation equations are

$$
\boldsymbol{\nabla} \cdot \boldsymbol{v}=0, \quad \partial \boldsymbol{v} / \partial t-\boldsymbol{v} \times \boldsymbol{\nabla} \times \boldsymbol{v}=-\nabla p+C_{g} \Delta \boldsymbol{v}
$$

if $(x, y) \in \Sigma$ and $-1<z<f$, with the boundary conditions

$$
\begin{gathered}
\boldsymbol{v}=\mathbf{0} \quad \text { if } z=-1 \text { or if }(x, y) \in \partial \Sigma, \quad f=0 \quad \text { if }(x, y) \in \partial \Sigma, \\
\boldsymbol{v} \cdot \boldsymbol{n}=(\partial f / \partial t)\left(\boldsymbol{e}_{z} \cdot \boldsymbol{n}\right),\left[\left(\boldsymbol{\nabla} \boldsymbol{v}+\boldsymbol{\nabla} \boldsymbol{v}^{\top}\right) \cdot \boldsymbol{n}\right] \times \boldsymbol{n}=\mathbf{0}, \quad \text { if } z=f, \\
p-|\boldsymbol{v}|^{2} / 2-(1-S) f+S \boldsymbol{\nabla} \cdot\left[\boldsymbol{\nabla} f /\left(1+|\boldsymbol{\nabla} f|^{2}\right)^{1 / 2}\right] \\
=C_{g}\left[\left(\boldsymbol{\nabla} \boldsymbol{v}+\boldsymbol{\nabla} \boldsymbol{v}^{\top}\right) \cdot \boldsymbol{n}\right] \cdot \boldsymbol{n}+4 \varepsilon \omega^{2} f \cos 2 \omega t \quad \text { if } z=f,
\end{gathered}
$$

and appropriate initial conditions. Here $p\left(=\right.$ pressure $+|\boldsymbol{v}|^{2} / 2+(1-S) z+$ $\left.4 \varepsilon \omega^{2} z \cos 2 \omega t\right)$ is a modified pressure, $v$ is the velocity, $f$ is the vertical deflection of the free surface, $\boldsymbol{n}$ is the outward unit normal to the free surface, $\partial \Sigma$ stands for the boundary of the container cross-section $\Sigma$, and $e_{z}$ is the upward unit vector. The real parameters $\varepsilon>0$ and $2 \omega$ are the forcing amplitude and frequency, $C_{g}=\nu /\left(g \ell^{3}+\sigma \ell / \rho\right)^{1 / 2}$ (with $\nu=$ kinematic viscosity) is a capillary-gravity number and $S=\sigma /\left(\sigma+\rho g \ell^{2}\right)$ is a gravity-capillary balance parameter. These are related to the usual capillary number $C=\nu \sqrt{\rho /(\sigma \ell)}$ and Bond number $B=\rho g \ell^{2} / \sigma$ as $C_{g}=C /(1+B)^{1 / 2}$ and $S=1 /(1+B)$.

Although the CASF equations below apply in a wider parameter range, for simplicity we consider the (nearly-inviscid, nearly-resonant, weakly-nonlinear) distinguished limit

$$
|\omega-\Omega| \sim C_{g}^{1 / 2} \sim \varepsilon \ll 1,
$$

where $\Omega$ is an inviscid eigenfrequency of the linearized problem around the quiescent state. We assume that $\Omega$ has (algebraic and geometric) multiplicity $N \geq 1$. The situation $N>1$ is nongeneric in the absence of symmetry in the sense that it is of higher codimension. However, in the presence of symmetry multiple symmetry-related modes can be excited simultaneously. Thus we consider small perturbations that split the eigenfrequency $\Omega$ into $N$ independent ones, $\Omega_{1}, \ldots, \Omega_{N}$, which will be assumed to be such that

$$
\left|\Omega_{k}-\Omega\right| \sim \varepsilon \ll 1 \quad \text { for } k=1, \ldots, N .
$$

The role of the streaming flow can be anticipated by considering the linearized problem around the quiescent state, whose solutions are of the form $(\boldsymbol{u}, p, f)=$ 
$\mathrm{e}^{\lambda t}(\boldsymbol{V}, P, F)+$ c.c., where $(\boldsymbol{V}, P, F)$ is a nontrivial solution of

$$
\begin{gathered}
\boldsymbol{\nabla} \cdot \boldsymbol{V}=\mathbf{0}, \quad \lambda \boldsymbol{V}=-\boldsymbol{\nabla} P+C_{g} \Delta \boldsymbol{V}, \quad \text { if }(x, y) \in \Sigma,-1<z<0, \\
\boldsymbol{V}=\mathbf{0} \text { if } z=-1 \text { or if }(x, y) \in \partial \Sigma, \quad F=0 \quad \text { if }(x, y) \in \partial \Sigma, \\
\boldsymbol{V} \cdot \boldsymbol{e}_{z}=\lambda F, \quad\left[\left(\boldsymbol{\nabla} \boldsymbol{U}+\boldsymbol{\nabla} \boldsymbol{U}^{\top}\right) \cdot \boldsymbol{e}_{z}\right] \times \boldsymbol{e}_{z}=\mathbf{0}, \\
P-(1-S) F+S \Delta F=C_{g}\left[\left(\boldsymbol{\nabla} \boldsymbol{V}+\boldsymbol{\nabla} \boldsymbol{V}^{\top}\right) \cdot \boldsymbol{e}_{z}\right] \cdot \boldsymbol{e}_{z} \text { if } z=0 .
\end{gathered}
$$

In the nearly-inviscid limit, $C_{g} \rightarrow 0$, this problem exhibits two kinds of nearlymarginal (with a small growth rate) modes. Either (i) $\lambda= \pm \mathrm{i} \Omega+O\left(\sqrt{C_{g}}\right)$, with $|\Omega| \sim|\boldsymbol{V}| \sim|P| \sim|F| \sim 1$ outside some viscous boundary layers of thickness $O\left(\sqrt{C_{g}}\right)$ attached to the (solid and free) boundary; these are the well-known surface (or nearly-inviscid) modes associated with the surface waves. Or (ii) $\lambda$ is real, negative and such that $|\lambda| \sim|P| \sim|F| \sim C_{g} \ll 1,|\boldsymbol{V}| \sim 1$; these are the so-called hydrodynamic (or viscous) modes, and are the ones associated with the streaming flow. Note that the latter modes are non-oscillatory, exhibit small freesurface deflection (both in contrast to the surface modes) and, like the surface modes, are near-marginal (and thus easily excited). Consequently neither mode type can be ignored a priori in a weakly nonlinear theory; the viscous modes may be ignored only in a strictly linear theory of the free surface motion. However, these modes, whose presence was recognized already by Lamb [13], are usually also ignored in the weakly nonlinear theory. We demonstrate below that this is in general inconsistent.

\section{Coupled Amplitude-Mean Flow Equations}

In addition to the fast time variable $t \sim 1$, we consider the slow variable

$$
T=\varepsilon t \text {. }
$$

Since $C_{g} \ll 1$, the surface-waves are nearly-inviscid in the bulk (outside the above-mentioned viscous boundary layers), where we may write

$$
\begin{aligned}
& (\boldsymbol{v}, p, f)=\varepsilon^{1 / 2} \mathrm{e}^{\mathrm{i} \omega t} \sum_{k=1}^{N} A_{k}\left(\boldsymbol{V}_{k}, P_{k}, F_{k}\right)+\text { c.c. } \\
& +\varepsilon\left[\left(\boldsymbol{v}^{s}, 0,0\right)+\sum_{k, l=1}^{N} \bar{A}_{k} A_{l}\left(\boldsymbol{\nabla} H_{k l}, P_{k l}, F_{k l}\right)+O T\right]+\ldots
\end{aligned}
$$

Here $\left(\boldsymbol{V}_{k}, P_{k}, F_{k}\right)$ is the limit as $\Omega_{k} \rightarrow \Omega$ of the inviscid eigenfunction associated with the eigenfrequency $\Omega_{k}, A_{k}$ is the associated complex amplitude that, together with the streaming flow velocity $\boldsymbol{v}^{s}$, is independent of the fast time $t$, overbars and c.c. stand for the complex conjugate, and OT denotes oscillatory terms. In addition, we assume that the eigenmodes can be selected such that

$$
\int_{-1}^{0} \int_{\Sigma} \boldsymbol{V}_{k} \cdot \boldsymbol{V}_{l} d \boldsymbol{x}+\int_{\Sigma}\left[(1-S) \bar{F}_{k} F_{l}+S \nabla F_{k} \cdot \nabla F_{l}\right] d x d y=\delta_{k l}
$$

where $\delta_{k l}$ is the Kronecker delta. This assumption is made only to simplify the derivation of the CASF equations and is frequently satisfied in practice. It allows 
us to obtain the leading order approximation to the energy of the system as

$$
\mathcal{E}=\sum_{k=1}^{N}\left|A_{k}\right|^{2}
$$

Note that the mean flow velocity has been decomposed into two parts, namely (i) the inviscid mean flow velocity, $\sum \bar{A}_{k} A_{l} \nabla H_{k l}$, with the potential $H_{k l}$ given by

$$
\begin{aligned}
& \Delta H_{k l}=0 \text { if }(x, y) \in \Sigma \text { and }-1<z<0, \\
& H_{k l}=0 \text { if either } z=-1 \text { or }(x, y) \in \partial \Sigma, \\
& \partial H_{k l} / \partial z=-\mathrm{i} \tilde{\nabla} \cdot\left(\bar{F}_{k} \tilde{\boldsymbol{V}}_{l}+F_{l} \overline{\tilde{\boldsymbol{V}}}_{k}\right) \text { if } z=0,
\end{aligned}
$$

accounting for the normal component of the mean flow velocity at the unperturbed boundary, and (ii) the viscous mean flow velocity, or streaming flow velocity, required to be tangent to the unperturbed boundary $\left(\boldsymbol{v}^{s} \cdot \boldsymbol{e}_{z}=0\right.$ at $\left.z=0\right)$.

The evolution equations for the complex amplitudes and the streaming flow are obtained from the solvability conditions for the equations that result when the expansion (11) is substituted into (1) and into the boundary conditions (for the solution in the bulk) that result from matching conditions with the solution in the viscous boundary layers. This process is a natural extension to the multimode problem of the asymptotic derivation in [14]. The resonant oscillatory terms at $O\left(\varepsilon^{3 / 2}\right)$ yield

$$
\begin{aligned}
A_{k}^{\prime}(T)=-\left[\delta_{k}(1+\mathrm{i})+\mathrm{i} d_{k}\right] A_{k}+\mathrm{i} \sum_{l, m, n=1}^{N} \alpha_{k l m n} \bar{A}_{l} A_{m} A_{n} \\
+\mathrm{i} \sum_{l=1}^{N} \beta_{k l} \bar{A}_{l}-\mathrm{i} \Omega \sum_{l=1}^{N} \int_{-1}^{0} \int_{\Sigma} \boldsymbol{v}^{s} \cdot \boldsymbol{g}_{k l} d \boldsymbol{x} A_{l}
\end{aligned}
$$

for $k=1, \ldots, N$, and from the non-oscillatory terms, at orders $\varepsilon$ and $\varepsilon^{2}$, one obtains

$$
\begin{gathered}
\boldsymbol{\nabla} \cdot \boldsymbol{v}^{s}=0 \\
\partial \boldsymbol{v}^{s} / \partial T-\left[\boldsymbol{v}^{s}+\sum_{k, l=1}^{N} \bar{A}_{k} A_{l}\left(\boldsymbol{\nabla} H_{k l}-\boldsymbol{g}_{k l}\right)\right] \times\left(\boldsymbol{\nabla} \times \boldsymbol{v}^{s}\right)=-\boldsymbol{\nabla} q^{m}+\varepsilon D \Delta \boldsymbol{v}\left({ }^{(s}, 9\right)
\end{gathered}
$$

for $(x, y) \in \Sigma,-1<z<0$, with the boundary conditions

$$
\begin{aligned}
& \boldsymbol{v}^{s}=\sum_{k, l=1}^{N} \bar{A}_{k} A_{l} \boldsymbol{\varphi}_{k l}^{1} \text { if } z=-1 \text { or if }(x, y) \in \partial \Sigma, \\
& \boldsymbol{v}^{s} \cdot \boldsymbol{e}_{z}=0, \quad \partial \tilde{\boldsymbol{v}}^{s} / \partial z=\sum_{k, l=1}^{N} \bar{A}_{k} A_{l} \boldsymbol{\varphi}_{k l}^{2} \text { at } z=0 .
\end{aligned}
$$

Here and hereafter the tildes stand for the horizontal projection (of vectors or operators) and the vectors $\varphi_{k l}^{1}$ and $\varphi_{k l}^{2}$ are tangent to the unperturbed boundary and depend on the position along it; these vectors, omitted here in the interests of brevity, can be written as hermitian bilinear, differential operators on $\left(\boldsymbol{V}_{k}, \boldsymbol{V}_{l}\right)$, obtained from the analysis of the boundary layers mentioned above. In addition, for all $k, l, m, n$,

$$
\begin{gathered}
\delta_{k}=\mu_{k} C_{g}^{1 / 2} / \varepsilon, \quad d_{k}=\left(\omega-\Omega_{k}\right) / \varepsilon, \quad D=C_{g} / \varepsilon^{2}, \\
\alpha_{k l m n}-\alpha_{k l n m}=\alpha_{k l m n}+\alpha_{l k m n}-\alpha_{m n l k}-\alpha_{n m l k}=0, \\
\boldsymbol{g}_{k l}=\mathrm{i} \Omega^{-1} \nabla \times\left(\overline{\boldsymbol{V}}_{k} \times \boldsymbol{V}_{l}\right) .
\end{gathered}
$$


Some remarks concerning the CASF equations above are now in order:

a. The amplitude equations (17) include terms accounting for inertia, viscous damping, detuning, cubic nonlinearity, parametric forcing and coupling with the streaming flow. Departure from the degenerate, multiple eigenvalue case is incorporated only in the detuning terms, a consistent procedure at leading order. Likewise, the viscous effects are only present explicitly in the damping term. Consequently, the cubic terms are conservative, and hence do not affect the energy of the system, given by (13), at leading order. The energy evolves according to

$$
d \mathcal{E} / d T=-2 \sum_{k=1}^{N} \delta_{k}\left|A_{2}\right|^{2}+\left[\sum_{k l=1}^{N} \mathrm{i} \beta_{k l} \bar{A}_{k} \bar{A}_{l}+\text { c.c. }\right],
$$

a result that follows on multiplying (17) by $\bar{A}_{k}$, adding the complex conjugate, adding up the results for $k=1, \ldots, N$, and using (23)-(24). This equation also shows that the term accounting for the coupling with the streaming flow is also conservative at leading order, which is not surprising since the unscaled kinetic energy of the streaming flow is of order $\varepsilon^{2}$, while that of the surface waves is of order $\varepsilon$.

$b$. The new term accounting for the coupling with the streaming flow is of the same order as the usual cubic nonlinear terms and thus, except for some special cases in which this term vanishes, it is inconsistent to neglect this term and retain only the cubic terms, as frequently done in the literature. Nonetheless, if the system is initially at rest, the total streaming flow momentum (and hence the coupling term) can remain small for a long time, until the momentum diffuses (or is convected) from the boundary into the bulk; see remark e below.

$c$. In the generic case $N=1$ the eigenfrequency $\Omega$ is algebraically simple, the phases of the eigenfunctions are constant, $\overline{\boldsymbol{V}}_{1}$ and $\boldsymbol{V}_{1}$ are colinear and (see (24)) $\boldsymbol{g}_{11} \equiv \mathbf{0}$. In this case the term accounting for the coupling with the streaming flow in the (one) amplitude equation (17) vanishes identically and the evolution of $A_{1}$ decouples from the streaming flow. Thus coupling requires the presence of at least two modes, as anticipated in $\S 1$.

$d$. The analysis above of the mean flow has been made for convenience in terms of the Eulerian velocity. This is just the average velocity on the fast timescale $t \sim 1$ and according to (11) is given by $\varepsilon\left(\boldsymbol{v}^{s}+\sum \bar{A}_{k} A_{l} \nabla H_{k l}\right)$ at leading order. The mass transport, or Lagrangian, velocity $[6,15]$ is

$$
\boldsymbol{v}^{m t}=\varepsilon\left(\boldsymbol{v}^{s}+\sum_{k, l=1}^{N} \bar{A}_{k} A_{l} \nabla H_{k l}\right)+\boldsymbol{v}^{S d}
$$

at leading order, and is associated with the averaged trajectories of material elements; the difference between them is the Stokes drift, and is given by

$$
\boldsymbol{v}^{S d}=-\varepsilon \sum_{k, l=1}^{N} \bar{A}_{k} A_{l} \boldsymbol{g}_{k l}
$$


at leading order, where $\boldsymbol{g}_{k l}$ is again given by (24). Note that both the inviscid mean flow and the Stokes drift are slaved to the surface waves, in contrast to the streaming flow. The mass transport velocity is relevant for comparison with flow visualizations (with an exposure time large compared to the forcing period) and, more generally, for analyzing the transport (and mixing) of passive scalars $[16,17]$. When the streaming flow is ignored this is usually because it is assumed that it is small compared to the Stokes drift [16]. However, except in some special cases (see below), this assumption is incorrect, as seen from (26)-(27). The normal component of the Eulerian mean-flow velocity at $z=0$ is non-zero in general, but this does not lead to any mass transport across the unperturbed free surface. To see this we must consider the mass transport velocity (26). Eqs. (16) and (24) and standard formulas from vector analysis show readily that $\nabla H_{k l} \cdot e_{z}=g_{k l} \cdot e_{z}$ at $z=0$, from which the result $\boldsymbol{v}^{m t} \cdot \boldsymbol{e}_{z}=0$ at $z=0$ follows at leading order on using (26)-(27).

$e$. The momentum equation (20) includes a non-standard term proportional to both the Lagrangian velocity and the Eulerian vorticity. This term is called the vortex force and does not force any flow by itself (it vanishes if $\boldsymbol{v}^{s}=0$ ) but can enhance or inhibit the effect of the remaining forcing terms; in fact this term can destabilize some shear streaming flows produced by water waves, like those associated with Langmuir circulations in ocean and lakes, see [15] and references therein. Moreover, we have included a $O(\varepsilon)$ viscous term even though it is of higher order because it is required to satisfy the boundary conditions (20)-(21) that force the streaming flow. Since $\varepsilon \ll 1$, we must expect thin viscous boundary layers attached to the solid walls and the free surface, with thickness $\varepsilon^{1 / 2}$ and $\varepsilon^{1 / 3}$, respectively. According to (5) these are thicker than the boundary layers mentioned in $\S 3$ that lead to the boundary conditions (20)-(21), as required for consistency of the analysis. Note also that we must expect (at least) two timescales in the analysis of the CASF equations, namely a convective timescale $T \sim 1$ and a viscous timescale, $T \sim \varepsilon^{-1}$, which can lead to extremely slow dynamics.

$f$. According to (22a), the damping rate at leading order of the $k$-th mode is $\mu_{k} C_{g}^{1 / 2}>0$, and results from viscous dissipation in the boundary layers mentioned above. Nevertheless, if the contact line is fixed, as we are assuming here, the coefficient $\mu_{k}$ is quite small for all but the first few low order modes [18] and (22a) must be replaced by $\delta_{k}=\left(\mu_{k} C_{g}^{1 / 2}+\mu_{k}^{\prime} C_{g}\right) / \varepsilon$. If, in addition, $C_{g}$ is not too small, then $\delta_{k}$ is in practice of order $C_{g} / \varepsilon$ and $\delta_{k} \sim 1$ requires that $C_{g} \sim \varepsilon$. Then $\varepsilon D \sim 1$ and the two timescales mentioned above are no longer distinct.

\section{Double-Mode Interaction in Almost-Square Containers}

Let us assume now that the cross-section of the cylinder is a rectangle that is close to the square

$$
\Sigma:|x|<L / 2, \quad|y|<L / 2,
$$

that the inviscid eigenfrequency $\Omega$ is algebraically double, and that the associated eigenfunctions are $\left(\boldsymbol{V}_{1}, P_{1}, F_{1}\right)$ such that $F_{1}$ is even in the $x$-direction 
and odd in the $y$-direction and $\left(\boldsymbol{V}_{2}, P_{2}, F_{2}\right)$, obtained from $\left(\boldsymbol{V}_{1}, P_{1}, F_{1}\right)$ by a reflection in the diagonal of the square. See $[9,10]$ and $[10,19,20,21,22,23]$ for experimental and theoretical analyses of this problem, all of them ignoring the streaming flow. Note that we are in the situation considered in $\S 2-\S 3$, with $N=2$. After rescaling, the CASF equations (17)-(21) become

$$
\begin{array}{r}
A_{ \pm}^{\prime}(\tau)=-[1+\mathrm{i}(\Gamma \pm \Lambda)] A_{ \pm}+\mathrm{i}\left(\alpha_{1}\left|A_{ \pm}\right|^{2}+\alpha_{2}\left|A_{\mp}\right|^{2}\right) A_{ \pm}+\mathrm{i} \alpha_{3} \bar{A}_{ \pm} A_{+}^{2} \\
+\mathrm{i} \Upsilon \bar{A}_{ \pm} \pm \Omega \int_{-1}^{0} \int_{\Sigma} \boldsymbol{u} \cdot \boldsymbol{g} d \boldsymbol{x} A_{\mp}, \\
\boldsymbol{\nabla} \cdot \boldsymbol{u}=0
\end{array}
$$

if $(x, y) \in \Sigma$ and $-1<z<0$, with boundary conditions

$$
\begin{gathered}
\boldsymbol{u}=\left(\left|A_{+}\right|^{2}+\left|A_{-}\right|^{2}\right) \varphi_{1}+\left(\left|A_{+}\right|^{2}-\left|A_{-}\right|^{2}\right) \varphi_{2}+\left(\bar{A}_{+} A_{-}+A_{+} \bar{A}_{-}\right) \varphi_{3} \\
+\mathrm{i}\left(\bar{A}_{+} A_{-}-A_{+} \bar{A}_{-}\right) \varphi_{4} \text { if either } z=-1 \text { or }(x, y) \in \partial \Sigma, \\
\boldsymbol{u} \cdot \boldsymbol{e}_{z}=0, \quad \partial \tilde{\boldsymbol{u}} / \partial z=\mathrm{i}\left(\bar{A}_{+} A_{-}-A_{+} \bar{A}_{-}\right) \boldsymbol{\varphi}_{5} \quad \text { if } z=0
\end{gathered}
$$

where $A_{+}=A_{1}, A_{-}=A_{2}, \boldsymbol{u}=\boldsymbol{v}^{s}, \tau=T, \Gamma=\omega-\left(\Omega_{1}+\Omega_{2}\right) / 2$ and $A=$ $\left(\Omega_{1}-\Omega_{2}\right) / 2$ up to $O(1)$ scaling factors, and

$$
\boldsymbol{H}=\mathrm{i}\left(\bar{A}_{+} A_{-}-A_{+} \bar{A}_{-}\right) \boldsymbol{h}, \quad \boldsymbol{G}=\mathrm{i}\left(\bar{A}_{+} A_{-}-A_{+} \bar{A}_{-}\right) \boldsymbol{g}, \quad R e \sim \varepsilon^{-1} \gg 1 .(34)
$$

Note that the detuning parameter $A$ is a rescaled measure of the departure of the cylinder cross-section from the square (28). The presence of this parameter is the only manifestation of the departure from the square cross-section, the remaining parameters and functions being calculated for the square case. Thus if $A=0$ the CASF equations are invariant under the actions

$$
\begin{array}{lr}
x \rightarrow-x, & A_{+} \rightarrow-A_{+}, \quad \boldsymbol{u} \equiv\left(u_{1}, u_{2}, u_{3}\right) \rightarrow\left(-u_{1}, u_{2}, u_{3}\right), \\
x \leftrightarrow y, & A_{+} \leftrightarrow A_{-}, \quad\left(u_{1}, u_{2}, u_{3}\right) \leftrightarrow\left(u_{2}, u_{1}, u_{3}\right),
\end{array}
$$

which generate a group that is isomorphic to the symmetry group $D_{4}$ of a square. Invariance of (29)-(33) under this group requires that (i) $\boldsymbol{h}, \boldsymbol{g}, \boldsymbol{\varphi}_{4}$ and $\boldsymbol{\varphi}_{5}$ be invariant under the actions

$$
\begin{aligned}
& x \rightarrow-x, \quad\left(u_{1}, u_{2}, u_{3}\right) \rightarrow\left(u_{1},-u_{2},-u_{3}\right), \\
& x \leftrightarrow y, \quad\left(u_{1}, u_{2}, u_{3}\right) \rightarrow\left(-u_{2},-u_{1},-u_{3}\right),
\end{aligned}
$$

(ii) $\varphi_{1}$ be invariant under $(35)$-(36), (iii) $\varphi_{2}$ be invariant under $x$ - and $y$ reflections and under the action (37) and (iv) $\varphi_{3}$ be invariant under reflections in the diagonals of the square and under the action (38).

\subsection{Reflection Symmetries and Timescales; Qualitative Comparison with Experiments}

Since $g$ is invariant under (37)-(38), the term describing the coupling with the streaming flow in (29) vanishes if the solution exhibits at least one of the reflection symmetries of the group $D_{4}$ generated by (35)-(36) (at each value of $\tau$ if 
the solution is time-dependent). Now we must take into account the symmetry properties of the container cross-section.

$A$. For squares $(\Lambda=0)$ there are three types of primary branches (all consisting of steady states), bifurcating from the flat state $\left(A_{+}=A_{-}=0\right)$ at $\Upsilon=\sqrt{1+\Gamma^{2}}$ : (i) pure states, of the form $A_{+} \neq 0, A_{-}=0$ and $A_{+}=0$, $A_{-} \neq 0$, (ii) standing mixed states of the form $A_{+}=A_{-}$and $A_{+}=-A_{-}$and (iii) progressive mixed states of the form $A_{+}=\mathrm{i} A_{-}$and $A_{+}=-\mathrm{i} A_{-}$. The first two ones (the only ones encountered in [9]) are reflection-symmetric and thus do not involve the streaming flow. However, their stability properties do in general involve this flow, which could explain the quantitative differences encountered in [9] (see also [23]) between experiment and theory that ignored the streaming flow. The progressive mixed (steady) states exhibit an apparent rotation and have nonzero angular momentum, as explained in [10]; they do not possess any of the reflection symmetries in the group $D_{4}$, and thus the streaming flow (which also exhibits an apparent rotation, and has a nonzero angular momentum and vertical vorticity) affects not only their stability properties but also their shape. Note that these mixed modes are the ones for which the discrepancy between experimental observations and the theory for square cells by Feng and Sethna $[10$, Fig.5] is the largest.

$B$. For rectangles $(A \neq 0)$ the $C A S F$ equations are no longer invariant under the group $D_{4}$, but only under the smaller group $D_{2}$ generated by (35) and

$$
y \rightarrow-y, \quad A_{-} \rightarrow-A_{-}, \quad \boldsymbol{u} \equiv\left(u_{1}, u_{2}, u_{3}\right) \rightarrow\left(u_{1},-u_{2}, u_{3}\right)
$$

There are now only two primary branches of pure steady states, and the mixed steady states appear through secondary bifurcations. Fot this system Simonelli and Gollub [9] report seeing several types of interesting time-dependent states (some extremely slow, see below) that are not reflection-symmetric (at each $\tau$ ) and thus should involve the streaming flow in an essential way. Thus far all attempts to explain these states using theory that neglects the presence of streaming flow have failed completely; the observations [9] constitute therefore a demanding test for any theory. Feng and Sethna's [10] theory compares well with the observations for the reflection-symmetric states (Fig. 6) but fails completely [10, Fig.6] for the steady progressive mixed states that should involve streaming flow. For example, where the theory predicted steady progressive mixed states Feng and Sethna observed in their experiments some extremely slow, amplitude modulated, rotating waves whose apparent chaotic nature could not be ascertained because of the extremely slow time scales involved.

In connection with the slow phenomena mentioned above, we note that the conclusion $R e \sim \varepsilon^{-1} \gg 1$ (see (34)) implies that the CASF equations (29)(33) exhibit dynamics on two distinct timescales ( 33 , remark $e$ ), namely $\tau \sim 1$ and $\tau \sim \varepsilon^{-1} \gg 1$. If the observed dynamics occur on the latter timescale, the observations may be used to investigate the role of the streaming flow and of initial conditions, as we now discuss.

$C$. When the system is initially at rest, namely $\boldsymbol{u} \equiv \mathbf{0}$ at $\tau=0$, a $O(1)$ tangential velocity will appear in a thin viscous boundary layer near the boundary where the vorticity is non-zero (due to the boundary conditions (32)-(33)), 
but the total streaming flow momentum in the bulk will remain small for a long time, $\tau \sim R e^{-1}$, until it diffuses (and is convected) into the bulk from the boundary layers. During this transient the integral appearing in (29) remains small and the system approaches an attractor of the amplitude equations usually considered in the literature, namely those obtained when the streaming flow is ignored. But, after this long transient, the streaming flow comes into play and the solution converges to the true attractor of the full $C A S F$ system, frequently involving the streaming flow. Transients of this type have been reported in $[9$, $\S 7.1]$ and in related laterally vibrated systems [24, Fig.8], and attractors reached on the viscous timescale might be involved in the experiment in [10]. If the viscous timescale is longer than the time span of the experiment and the initial streaming velocity is appropriately small, the effect of the streaming flow will not manifest itself during the experiment. This fact could explain why some of the observations by Feng and Sethna [10] agreed with a weakly nonlinear theory that ignored streaming flow, while those by Simonelli and Gollub [9] did not; the cross-section of the container in the former case was more than three times larger and the viscous timescale therefore at least 10 times longer (of the order of several hours).

D.- If the initial streaming flow velocity is nonzero and satisfies the boundary conditions, the initial vorticity is also nonzero, and the streaming flow affects the dynamics of the surface waves from the very beginning. During an initial long transient on the convective timescale viscous diffusion in the momentum equation (31) can be ignored; the resulting system of simplified CASF equations can exhibit "attractors" that need not be close to the true attractors of the system, which will be reached only on the viscous timescale. Transients of this type might be responsible for the striking behavior reported in [9, Fig.16] and described as 'structural instability', namely, that in these long transients the behavior of the system depends strongly on the initial condition for the streaming flow velocity, which is not controlled in the experiments [9].

This work was supported by NASA (Grant NAG3-2152) and DGES (Grant PB97-0556).

\section{References}

1. M. Faraday, On the forms and states assumed by fluids in contact with vibrating elastic surfaces Phil. Trans. Roy. Soc. London 121 (1831), 319-340.

2. J. Miles and D. Henderson, Parametrically forced surface waves, Annu. Rev. Fluid Mech. 22 (1990), 143-165.

3. M.C. Cross and P.C. Hohenberg, Pattern formation outside of equilibrium Rev. Modern Phys. 65 (1993), 851-1112.

4. S. Fauve, Parametric instabilities, in Dynamics of Nonlinear and Disordered Systems, G. Martínez-Mekler and T.H. Seligman eds., World Scientific, pp.67-115, 1995.

5. H. Schlichting, Berechnung ebener periodischer Grenzschichtströmungen, Phys. Z. 33 (1932), 327-335. 
6. M.S. Longuet-Higgins, Mass transport in water waves, Phil. Trans. Roy. Soc. A 245 (1953), 535-581.

7. M. Higuera, J.A. Nicolás and J.M. Vega, Coupled amplitude-streaming flow equations for the evolution of counter-rotating, nearly-inviscid surface waves in finite axisymmetric geometries. Preprint, 2000.

8. F. Mashayek and N. Ashgriz, Nonlinear oscillations of drops with internal circulation, Phys. Fluids 10 (1998), 1071-1082.

J. Fluid Mech. 158(1985)381-398.

9. F. Simonelli and J.P. Gollub, Surface wave mode interactions: effects of symmetry and degeneracy, J. Fluid Mech. 199 (1989), 471-494.

10. Z.C. Feng and P.R. Sethna, Symmetry breaking bifurcations in resonant surface waves, J. Fluid Mech. 199 (1989), 495-518.

11. G.W. Young and S.H. Davis, A plate oscillating across a liquid interface: effects of contact-angle hystheresis, J. Fluid Mech. 174 (1987), 327-356.

12. J. Bechhoefer, V. Ego, S. Manneville and B. Johnson, An experimental study of the onset of parametrically pumped surface waves in viscous fluids, J. Fluid Mech. 288 (1995), 325-350.

13. H. Lamb, Hydrodynamics, Cambridge University Press, 1932.

14. J.A. Nicolás and J.M. Vega, Weakly nonlinear oscillations of axisymmetric liquid bridges, J. Fluid Mech. 328 (1996), 95-128.

15. A.D.D. Craik, Wave Interactions and Fluid Flows, Cambridge Univ. Press, 1985.

16. M. Umeki, Particle transport by angular momentum on three-dimensional standing surface waves, Phys. Rev. Lett. 67 (1991), 2650-2653.

17. Z.C. Feng and S. Wiggins, Fluid particle dynamics and Stokes drift in gravity and capillary waves generated by the Faraday instability, Nonlinear Dynamics 8 (1995), 141-160.

18. C. Martel, J.A. Nicolás and J.M. Vega, Surface-wave damping in a brimful circular cylinder, J. Fluid Mech. 360 (1998), 213-228. See also Corrigendum, 373 (1998), 379.

19. M. Silber and E. Knobloch, Parametrically excited waves in square geometry, Phys. Lett. A 137 (1989), 471-494.

20. M. Nagata, Nonlinear Faraday resonance in a box with a square base, J. Fluid Mech. 209 (1989), 265-284.

21. M. Nagata, Chaotic behavior of parametrically excited surface waves in square geometry, Eur. J. Mech. B/Fluids 10 (1991), 61-66.

22. M. Umeki, Faraday resonance in rectangular geometry, J. Fluid Mech. 227 (1991), 161-192.

23. D. Armbruster, J. Guckenheimer and S. Kim, Resonant surface waves in a square container, in Differential Equations and Computer Algebra, M. Singer ed., Academic Press, pp.61-76, 1991.

24. M. Funakoshi and S. Inoue, Surface waves due to resonant horizontal oscillation, J. Fluid Mech. 192 (1988), 219-247. 